\title{
Behaviour of a Shear-Wave at a Solid-Smectic Interface
}

\author{
B. C. Snow and I. W. Stewart \\ Department of Mathematics and Statistics, University of Strathclyde, \\ Glasgow, Scotland, United Kingdom
}

\begin{abstract}
Results of theoretical investigations into the behaviour of a shear wave at the boundary between an isotropic solid and a smectic A liquid crystal are presented. These results track the subsequent response of the smectic to the refracted wave. Using the techniques of Landau and Lifshitz for sound in isotropic fluids [1], we extend the results for smectic C by Gill and Leslie [2] and perform the analogous calculations for a sample of smectic A using the dynamic theory of Stewart [3]. These calculations enable a comparison between the results for smectic A and an extension, by the present authors, to the known results for smectic C.

Motivated by the work of Auernhammer, Brand, and Pleiner [4, 5], mechanisms for determining the impact of perturbations upon the modes of response behaviour will be analysed, with plots demonstrating the amplitudes of these waves relative to that of the incident wave displayed for a range of typical physical parameters characteristic to smectic $\mathrm{C}$ and smectic A.
\end{abstract}

\section{Introduction}

It is well known that smectic liquid crystals have the potential for much faster switching than their nematic counterparts. As was remarked by Gill and Leslie [2], induced flow can play a major role in the dynamics of such switching, and it is therefore of great interest to develop a comprehensive understanding of how smectics behave when subjected to a variety of perturbations and induced flow profiles.

The analysis presented herein will consider a perturbation to a sample of bookshelf aligned smectic A (SmA) induced by a shear wave incident at the plane interface between the smectic and an isotropic elastic solid such that the wave first propagates through the solid then undergoes a reflection and refraction on contact with the interface, as shown in Fig. 1.

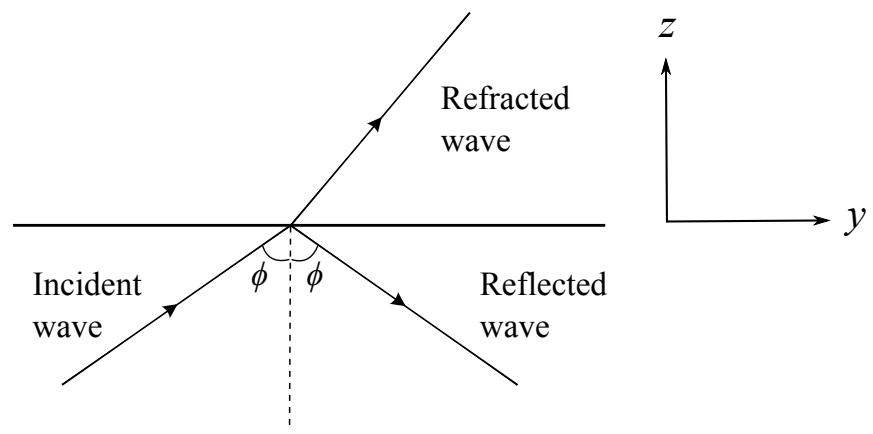

Figure 1: On contact with the interface, the incident wave is both reflected and refracted. The region $z<0$ is occupied by an isotropic elastic solid, while the region $z>0$ contains bookshelf aligned SmA. 
Section 2 will provide a brief outline of Stewart's dynamic theory for SmA [3]. Section 3 will provide, in an analogous manner to that adopted by Gill and Leslie [2] for smectic C (SmC), derivations of the dispersion relations for the reflected and refracted waves, together with the consequent interfacial conditions to be utilised in the analysis. A brief summary of the results from [2] will be presented in Section 4, before deriving, in Section 5, expressions that relate the amplitudes of the reflected and refracted waves in terms of the incident wave amplitude and material parameters that characterise $\mathrm{SmA}$ and $\mathrm{SmC}$, the latter being a novel extension to the work in [2]. Section 5 concludes with plots which demonstrate how the amplitudes of the refracted waves vary with the incident angular frequency of the incident wave. The paper will close with a summary and discussion of the main results and the potential influence on further work.

\section{Continuum Theory for Smectic A Liquid Crystals}

It is known that nematic liquid crystals consist of rod-like molecules that tend to align parallel to each other along some common preferred direction indicated by the unit vector $\boldsymbol{n}$, called the director. The SmA liquid crystal phase occurs when the constituent molecules are arranged in layers, where the director is, on average, generally aligned perpendicular to the local layer structure and is parallel to the local layer normal. In $\mathrm{SmC}$ liquid crystals the director is tilted at an angle $\theta$ relative to the layer normal; $\theta$ is usually temperature dependent and is called the smectic cone angle. The director $\boldsymbol{n}$ continues to be defined as the average direction of the molecular alignment and, from the physical point of view, $\boldsymbol{n}$ and $\boldsymbol{- n}$ are indistinguishable. It is the SmA phase that is the main concern here, although a comparison with results for $\mathrm{SmC}$ will also be made.

We now summarise the continuum theory in [3], in which $\boldsymbol{n}$ and $\boldsymbol{a}$ are allowed to separate, as considered by Ribotta and Durand [6]. The standard suffix notation for Cartesian vectors and tensors [7] is employed. SmA is described by two unit vectors: the layer normal $\boldsymbol{a}$ and the director $\boldsymbol{n}$. The layer normal is perpendicular to the plane of the smectic layers, and it is conveniently defined via a scalar function $\Phi(x, y, z, t)$ such that

$$
\boldsymbol{a}=\frac{\nabla \Phi}{|\nabla \Phi|}, \quad \text { i.e., } \quad a_{i}=\frac{\Phi_{, i}}{|\nabla \Phi|}
$$

from which is it clear that $\boldsymbol{a}$ is a unit vector by its definition. As mentioned, $\boldsymbol{n}$ is also constrained to be a unit vector, so that

$$
\boldsymbol{n} \cdot \boldsymbol{n}=n_{i} n_{i}=1
$$

We will be concerned with samples which are both isothermal and incompressible (in the classical sense); the latter of these requires that the velocity $\boldsymbol{v}$ at any point in the smectic satisfies

$$
v_{i, i}=0 .
$$

The balance of linear momentum takes the form

$$
\rho \dot{v}_{i}=\rho f_{i}-\tilde{p}_{, i}+\tilde{g}_{j} n_{j, i}+G_{j} n_{j, i}+|\nabla \Phi| a_{i} J_{j, j}+\tilde{t}_{i j, j}
$$

with $\rho$ denoting the density, $f_{i}$ the external body force per unit mass, $\tilde{p}=p+w_{A}$, where $p$ is the pressure and $w_{A}$ is the elastic energy density given by [8]

$$
\begin{aligned}
w_{A} & =\frac{1}{2} K_{1}^{a}(\nabla \cdot \boldsymbol{a})^{2}+\frac{1}{2} K_{1}^{n}\left(\nabla \cdot \boldsymbol{n}-s_{0}\right)^{2}+\frac{1}{2} K_{2} \nabla \cdot[(\boldsymbol{n} \cdot \nabla) \boldsymbol{n}-(\nabla \cdot \boldsymbol{n}) \boldsymbol{n}] \\
& +\frac{1}{2} B_{0}|\nabla \Phi|^{-2}(1-|\nabla \Phi|)^{2}+\frac{1}{2} B_{1}\left[1-(\boldsymbol{n} \cdot \boldsymbol{a})^{2}\right] \\
& +B_{2}(\nabla \cdot \boldsymbol{n})\left(1-|\nabla \Phi|^{-1}\right),
\end{aligned}
$$

where $K_{1}^{a}, K_{1}^{n}$, and $K_{2}$ are elastic constants characterising, respectively, the deformation due to bending of the smectic layers, the director splay (with $s_{0}$ denoting the spontaneous splay), and 
the director saddle-splay; $B_{0}$ is the layer compression constant, $B_{1}$ is the constant attributed to coupling between $\boldsymbol{n}$ and $\boldsymbol{a}$, and $B_{2}$ characterises the energy due to the coupling between splay and layer compression. The superposed dot represents the usual material time derivative. The term $\tilde{t}_{i j}$ is the viscous stress with components

$$
\begin{aligned}
\tilde{t}_{i j} & =\alpha_{1}\left(n_{k} A_{k l} n_{l}\right) n_{i} n_{j}+\alpha_{2} N_{i} n_{j}+\alpha_{3} n_{i} N_{j}+\alpha_{4} A_{i j}+\alpha_{5}\left(n_{j} A_{i k} n_{k}+n_{i} A_{j k} n_{k}\right) \\
& +\left(\alpha_{2}+\alpha_{3}\right) n_{i} A_{j k} n_{k}+\tau_{1}\left(a_{k} A_{k l} a_{l}\right) a_{i} a_{j}+\tau_{2}\left(a_{i} A_{j k} a_{k}+a_{j} A_{i k} a_{k}\right) \\
& +\kappa_{1}\left(a_{i} N_{j}+N_{i} a_{j}+n_{i} A_{j k} a_{k}-n_{j} A_{i k} a_{k}\right)+\kappa_{2}\left(n_{k} A_{k l} n_{l}\right)\left(n_{i} a_{j}+a_{i} n_{j}\right) \\
& +\kappa_{3}\left[\left(n_{k} A_{k l} n_{l}\right) a_{i} a_{j}+\left(a_{k} A_{k l} a_{l}\right) n_{i} n_{j}\right] \\
& +\kappa_{4}\left[2\left(n_{k} A_{k l} a_{l}\right) n_{i} n_{j}+\left(n_{k} A_{k l} n_{l}\right)\left(a_{i} n_{j}+n_{i} a_{j}\right)\right] \\
& +\kappa_{5}\left[2\left(n_{k} A_{k l} a_{l}\right) a_{i} a_{j}+\left(a_{k} A_{k l} a_{l}\right)\left(n_{i} a_{j}+a_{i} n_{j}\right)\right] \\
& +\kappa_{6}\left(n_{j} A_{i k} a_{k}+n_{i} A_{j k} a_{k}+a_{i} A_{j k} n_{k}+a_{j} A_{i k} n_{k}\right) .
\end{aligned}
$$

The coefficients on the right-hand side of (2.6) are dynamic viscosities, while $A_{i j}$ denotes the usual rate of strain tensor, with components

$$
A_{i j}=\frac{1}{2}\left(v_{i, j}+v_{j, i}\right),
$$

and $\boldsymbol{N}$ is the co-rotational time flux, defined as $N_{i}=\dot{n}_{i}-W_{i j} n_{j}$, where $W_{i j}$ is the vorticity tensor whose components are given by

$$
W_{i j}=\frac{1}{2}\left(v_{i, j}-v_{j, i}\right)
$$

The term $\tilde{g}_{i}$ is a dynamic contribution given by

$$
\tilde{g}_{i}=-\left(\alpha_{3}-\alpha_{2}\right) N_{i}-\left(\alpha_{2}+\alpha_{3}\right) A_{i j} n_{j}-2 \kappa_{1} A_{i j} a_{j}
$$

and $G_{i}$ is the generalised external body force, which is related to the external body moment $K_{i}$ per unit mass via

$$
\rho K_{i}=\epsilon_{i j k} n_{j} G_{k} .
$$

The vector $\boldsymbol{J}$ is the negative of the permeative force $\boldsymbol{\tau}$, and has components as

$$
J_{i}=-\frac{\partial w_{A}}{\partial \Phi_{, i}}+\frac{1}{|\nabla \Phi|}\left[\left(\frac{\partial w_{A}}{\partial a_{j, k}}\right)_{, k}-\frac{\partial w_{A}}{\partial a_{j}}\right]\left(\delta_{j i}-a_{j} a_{i}\right),
$$

The balance of angular momentum may be expressed in the form

$$
\left(\frac{\partial w_{A}}{\partial n_{i, j}}\right)_{, j}-\frac{\partial w_{A}}{\partial n_{i}}+\tilde{g}_{i}+G_{i}=\lambda n_{i}
$$

where $\lambda$ is a Lagrange multiplier arising from the unit vector constraint on $\boldsymbol{n}$ as given in equation (2.2), which can generally be either evaluated or eliminated on taking the scalar product of (2.10) with $\boldsymbol{n}$. The permeation equation, which describes permeative flow between the smectic layers, is

$$
\dot{\Phi}=-\lambda_{p} J_{i, i}
$$

where $\lambda_{p} \geq 0$ is the permeation coefficient.

\section{Reflection and Refraction of the Shear Wave at the Solid-Smectic A Interface}

Consider Fig. 2, which depicts a plane interface between an isotropic elastic solid and a sample of bookshelf aligned SmA. We may take the interface to lie parallel to the $x y$-plane and, following [2], 




Figure 2: Problem set-up for "bookshelf" SmA.

assume the solid and smectic to be unbounded in space. Referred to the geometry of Fig. 1, the initially undistorted sample of bookshelf aligned SmA will have its configuration described by

$$
\boldsymbol{n}=\boldsymbol{n}_{\mathbf{0}}=(0,1,0), \Phi=\Phi_{0}=y .
$$

This initial configuration will be perturbed by an incident shear wave with displacement $\boldsymbol{u}=$ $\left(u_{x}, u_{y}, u_{z}\right)$ whose components are

$$
u_{x}=A \exp \{i[\omega t-k(y \sin \phi+z \cos \phi)]\}, u_{y}=u_{z}=0,
$$

where $A$ is the constant (real, without loss of generality) amplitude of the wave and $\phi$ is the constant angle of incidence (to the interface's normal); $\omega$ and $k$ denote the constant incident frequency and wave number, respectively. This displacement is required to satisfy the wave equation for an isotropic solid [9, p. 87]. For the displacement as given in (3.2), this leads to the relation

$$
\rho_{s} \omega^{2}=\mu_{s} k^{2}
$$

where $\mu_{s}$ and $\rho_{s}$ denote, respectively, the shear modulus (or bulk modulus) and density of the solid. It is natural to suppose that the displacement of the reflected wave $\boldsymbol{u}^{r}=\left(u_{x}^{r}, u_{y}^{r}, u_{z}^{r}\right)$ takes the form

$$
u_{x}^{r}=B \exp \{i[\omega t-k(y \sin \phi-z \cos \phi)]\}, u_{y}^{r}=u_{z}^{r}=0
$$

with $B$ a constant complex amplitude which will be determined below.

It is expected that the incident wave will induce a perturbation to the smectic. It will be assumed that this disturbance may cause small changes to the alignment of the constituent molecules (that is, to the director) and to the layer normal. The refracted wave velocity in the smectic is assumed to be of the form

$$
v_{x}=v \exp \{i[\omega t-k(y \sin \phi+q z)]\}, v_{y}=v_{z}=0,
$$

with $v, q \in \mathbb{C}$ to be determined below. Note that this form of $\boldsymbol{v}$ automatically satisfies the incompressibility condition $v_{i, i}=0$. The perturbed scalar $\Phi$ will be given by

$$
\Phi=y-u(y, z, t),
$$

for small perturbations $u=\hat{u} \exp \{i[\omega t-k(y \sin \phi+q z)]\}$ such that $|\hat{u}| \ll 1$. Then, to first order in $u$ and derivatives thereof,

$$
\boldsymbol{a}=\frac{\nabla \Phi}{|\nabla \Phi|}=\left(0,1,-u_{z}\right)
$$

Finally, the director $\boldsymbol{n}=\left(n_{x}, n_{y}, n_{z}\right)$ is perturbed so that

$$
n_{x}=n \exp \{i[\omega t-k(y \sin \phi+q z)]\}, n_{y}=1, n_{z}=0 .
$$

Substitution of (3.5) to (3.8) into the balance laws for linear and angular momentum (2.4) and (2.10) as well as the permeation equation (2.11) leads to the requirement $\hat{u} \equiv 0$. Therefore $\boldsymbol{a} \equiv(0,1,0)$ to first order. Further, two dispersion relations are identified:

$$
\begin{aligned}
{\left[2 i \rho \omega+k^{2}\left(\alpha_{4} q^{2}-\eta \sin ^{2} \phi\right)\right] v-2 \omega k\left(\alpha_{2}+\kappa_{1}\right) n \sin \phi } & =0 \\
\left(\alpha_{2}+\kappa_{1}\right) k v \sin \phi+\left[\left(\alpha_{2}-\alpha_{3}\right) \omega-B_{1}\right] n & =0
\end{aligned}
$$


where

$$
\eta=\alpha_{2}-\alpha_{4}-\alpha_{5}-\tau_{2}+2\left(\kappa_{1}-\kappa_{6}\right)
$$

upon identification of $\lambda$ as $B_{1}$ and with the pressure reducing to an arbitrary function of time $t$. Equations (3.9) and (3.10) furnish us with non-trivial solutions for $v$ and $n$ provided the relation

$$
\left[2 i \rho \omega+k^{2}\left(\alpha_{4} q^{2}-\eta \sin ^{2} \phi\right)\right]\left[\left(\alpha_{2}-\alpha_{3}\right) \omega-B_{1}\right]+2 \omega k^{2}\left(\alpha_{2}+\kappa_{1}\right)^{2} \sin ^{2} \phi=0
$$

is satisfied. Rearranging this for $q^{2}$ yields

$$
q^{2}=\beta_{1}-2 i \beta_{2},
$$

where $\beta_{1}$ and $-2 \beta_{2}$ are the real and imaginary parts of $q^{2}$, respectively, given by

$$
\beta_{1}=\left[\eta+\frac{2 \omega\left(\alpha_{2}+\kappa_{1}\right)^{2}}{B_{1}+\gamma_{1} \omega}\right] \sin ^{2} \phi, \quad \beta_{2}=\frac{\rho \xi}{\alpha_{4}},
$$

and we have introduced the notation

$$
\xi=\omega / k^{2}
$$

for later comparison with [2]. Equation (3.13) yields two solutions for $q$. To ensure bounded solutions, it is clear from the forms of the perturbed quantities that the root with negative imaginary part is required here. One therefore finds

$$
q=\chi-i \psi, \quad \psi>0
$$

where

$$
\chi=\frac{\beta_{2}}{\psi}, \quad \psi=\sqrt{\frac{1}{2}\left(\sqrt{\beta_{1}^{2}+4 \beta_{2}^{2}}-\beta_{1}\right)} .
$$

Note that normal incidence gives

$$
\left.q\right|_{\phi=0}=(1-i) \sqrt{\frac{\rho \xi}{\alpha_{4}}}
$$

\section{Boundary Conditions}

Continuity of velocity and surface traction are imposed at the interface. Using the above expressions for displacement in the solid of the incident and reflected waves in (3.2) and (3.4), respectively, combined with equation (3.5), continuity of velocity leads to the relation

$$
i \omega(A+B)=v .
$$

In the solid, the surface traction is required to satisfy the constitutive equations for isotropic elasticity [10, p. 115], so that continuity of surface traction at the interface imposes the requirement

$$
2 \mu_{s}(A-B) \cos \phi=\alpha_{4} q v \text {. }
$$

\section{The Smectic C Case}

Figure 3 depicts a set-up analogous to that considered above, but with a sample of $\mathrm{SmC}$ in place of the SmA. This is the geometry of Gill and Leslie's problem [2]. For an incident wave of the form given in (3.2), we obtain (3.3) as before and anticipate a reflected wave displacement identical to that given in (3.4) with $B$ now dependent on certain SmC material parameters, as established below.

Following the Leslie-Stewart-Nakagawa (LSN) description of SmC [11], Gill and Leslie allowed for perturbations to the $c$-director $\boldsymbol{c}=\left(c_{x}, c_{y}, c_{z}\right)$ of the form

$$
c_{x}=c \exp i[\omega t-k(y \sin \phi+q z)], c_{y}=\sin \theta, c_{z}=-\cos \theta,
$$




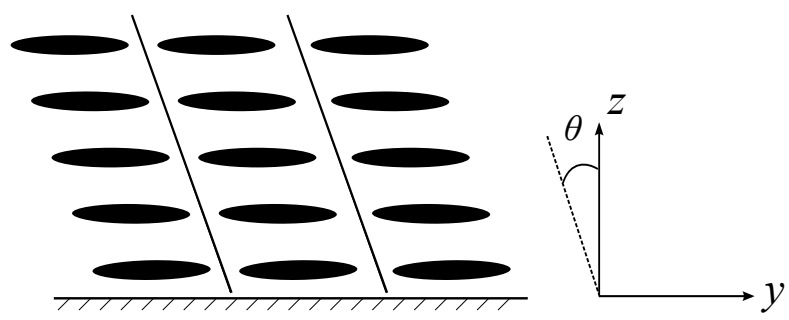

Figure 3: The analogous problem for SmC.

where $\theta$ denotes the usual smectic cone angle (see Fig. 3). (Note that setting $c_{x}=0$ in equation (4.1) yields the undistorted initial configuration as depicted in Fig. 3.). Substitution of (4.1) along with a velocity vector of the form given in (3.5) into the balance of linear and angular momentum equations for $\mathrm{SmC}$ (see [12, p. 295]) gives [2]

$$
\begin{gathered}
{\left[2 i \rho \omega+k^{2} \eta(q)\right] v-2 \omega k \nu(q) c=0,} \\
i k \nu(q) v-\left[2 i \lambda_{5} \omega+k^{2} \sigma(q)\right] c=0,
\end{gathered}
$$

leading to the analogue of condition (3.12) for non-trivial solutions:

$$
[\eta(q)+2 i \rho \xi]\left[\sigma(q)+2 i \lambda_{5} \xi\right]-2 i \xi \nu^{2}(q)=0 .
$$

In the above, $\eta(q)$ and $\nu(q)$ denote the somewhat unwieldy combinations of $\mathrm{SmC}$ viscosities

$$
\eta(q)=\eta_{1} q^{2}+\eta_{2} q \sin \phi+\eta_{3} \sin ^{2} \phi,
$$

where

$$
\begin{aligned}
& \eta_{1}=\eta_{11} \sin ^{2} \theta+\eta_{12} \sin (2 \theta)+\eta_{13} \cos ^{2} \theta, \\
& \eta_{2}=2 \eta_{12} \cos (2 \theta)+\left(\eta_{11}-\eta_{13}\right) \sin (2 \theta), \\
& \eta_{3}=\eta_{11} \cos ^{2} \theta-\eta_{12} \sin (2 \theta)+\eta_{13} \sin ^{2} \theta,
\end{aligned}
$$

with

$$
\begin{aligned}
& \eta_{11}=\mu_{0}+\mu_{2}-2 \lambda_{1}+\lambda_{4}, \\
& \eta_{12}=\tau_{1}+\tau_{2}-\tau_{5}-\kappa_{1}, \\
& \eta_{13}=\mu_{0}+\mu_{4}-2 \lambda_{2}+\lambda_{5},
\end{aligned}
$$

and

$$
\nu(q)=\nu_{1} q+\nu_{2} \sin \phi,
$$

where

$$
\begin{aligned}
& \nu_{1}=\left(\tau_{1}-\tau_{5}\right) \sin \theta-\left(\lambda_{2}-\lambda_{5}\right) \cos \theta, \\
& \nu_{2}=\left(\tau_{1}-\tau_{5}\right) \cos \theta+\left(\lambda_{2}-\lambda_{5}\right) \sin \theta .
\end{aligned}
$$

Also, the term $\sigma(q)$ denotes combination of SmC elastic constants, and is given by

$$
\sigma(q)=\sigma_{1} q^{2}+\sigma_{2} q \sin \phi+\sigma_{3} \sin ^{2} \phi,
$$

where (in the notation of Stewart [12, equations (6.15), (6.34), and (6.25)]),

$$
\begin{aligned}
& \sigma_{1}=K_{4} \sin ^{2} \theta+K_{7} \sin (2 \theta)+K_{3} \cos ^{2} \theta, \\
& \sigma_{2}=2 K_{7} \cos (2 \theta)+\left(K_{4}-K_{3}\right) \sin (2 \theta), \\
& \sigma_{3}=K_{4} \cos ^{2} \theta-K_{7} \sin (2 \theta)+K_{3} \sin ^{2} \theta .
\end{aligned}
$$


Equation (4.4) is quartic in $q$, so that there are four solutions, two of which are of physical relevance. These are [13]

$$
q_{1}=(1-i) \sqrt{\frac{\xi \Gamma_{1}}{\eta_{1} \sigma_{1}}}, \quad \text { and } \quad q_{2}=\epsilon-i \zeta, \quad \zeta>0
$$

where

$$
\epsilon=\frac{b_{2}}{\zeta}-\frac{\Gamma_{2} \sin \phi}{2 \Gamma_{1}}, \quad \zeta=\sqrt{\frac{1}{2}\left(\sqrt{b_{1}^{2}+4 b_{2}^{2}}-b_{1}\right)},
$$

with

$$
b_{1}=\left(\frac{\Gamma_{2}^{2}}{4 \Gamma_{1}^{2}}-\frac{\Gamma_{3}}{\Gamma_{1}}\right) \sin ^{2} \phi, \quad b_{2}=\frac{\rho \xi \lambda_{5}}{\Gamma_{1}}
$$

on setting

$$
\Gamma_{1}=\lambda_{5} \eta_{1}-\nu_{1}^{2}, \quad \Gamma_{2}=\lambda_{5} \eta_{2}-2 \nu_{1} \nu_{2}, \quad \Gamma_{3}=\lambda_{5} \eta_{3}-\nu_{2}^{2},
$$

upon making use of certain approximations, for instance $\rho \sigma_{1} \ll \Gamma_{1}$ [2]. Note that, at normal incidence, $q_{2}$ reduces to

$$
\left.q_{2}\right|_{\phi=0}=(1-i) \sqrt{\frac{\rho \xi \lambda_{5}}{\Gamma_{1}}},
$$

while $q_{1}$ shows no dependence upon the angle of incidence to first order. In this case, the Stokes layers [1, p. 84] for mode 1 and mode 2 are, respectively,

$$
\delta_{1}=\sqrt{\frac{\eta_{1} \sigma_{1}}{\omega \Gamma_{1}}}, \quad \delta_{2}=\frac{1}{k \zeta},
$$

the second of these reducing at normal incidence to

$$
\left.\delta_{2}\right|_{\phi=0}=\sqrt{\frac{\Gamma_{1}}{\rho \omega \lambda_{5}}} .
$$

As remarked by Gill [13], the dependence of $q_{1}$ on the elastic constants leads it to being regarded as an orientational mode relating to attenuation of reorientation of the $c$-director, while mode 2 is a hydrodynamic mode, characterising attenuation due to the diffusion of a vorticity. Note that the corresponding dependence of $\delta_{1}$ on $\sigma_{1}$ leads to the conclusion that $\delta_{1} \ll \delta_{2}$, and thus mode 2 will be dominant after a depth $\sim \delta_{1}$ into the smectic.

In what follows, it is the behaviour of mode 2 which will be of interest as it is this mode which is analogous to the SmA solution for $q$ at (3.16) in SmA. In this case, the depth of the Stokes layer is $\delta_{A}=1 / k \psi$, which reduces at normal incidence to

$$
\left.\delta_{A}\right|_{\phi=0}=\sqrt{\frac{\alpha_{4}}{\rho \omega}} .
$$

This is identical to the Stokes layer that occurs in oscillatory flow of SmA [14], and is analogous to that for isotropic fluids [1, p.84]. From this, it is clear that viscous behaviour is dominant in characterising the propagation of this disturbance.

It is worth remarking here that if one were to perform an analysis of a SmA sample whose layers were tilted relative to the interface, one would expect to find a second mode of propagation analogous to mode one in the case of SmC. While such an investigation is beyond the scope of the present paper, it is the intention of the present authors to consider this at a later date. 


\section{Interfacial Conditions}

As in Section 3, we require continuity of velocity, leading to the condition [2]

$$
i \omega(A+B)=v_{1}+v_{2},
$$

where $v_{1}$ and $v_{2}$ denote the velocity contributions to the refracted wave of mode 1 and mode 2 , respectively. Continuity of surface traction imposes the requirement

$$
2 \mu_{s}(A-B) \cos \phi=\eta_{1}\left(q_{1} v_{1}+q_{2} v_{2}\right)+\frac{1}{2} \eta_{2}\left(v_{1}+v_{2}\right) \sin \phi
$$

the latter reducing at normal incidence to

$$
2 \mu_{s}(A-B)=\eta_{1}\left(q_{1} v_{1}+q_{2} v_{2}\right) .
$$

Imposing strong anchoring at the boundary requires that

$$
c_{1}+c_{2}=0
$$

where $c_{1}$ and $c_{2}$ are the distortions of the $c$-director corresponding to mode 1 and mode 2 , respectively.

\section{Solutions for the Amplitudes: Comparison at Nor- mal Incidence}

We now present a comparison of the anticipated response behaviour of each of the smectics. For brevity, we will only outline the case of normal incidence $(\phi \equiv 0)$; a full account of the more general oblique incidence case is currently in preparation.

\section{Smectic A}

Equation (3.20) reduces at normal incidence to

$$
2 \mu_{s}(A-B)=\alpha_{4} q v
$$

which, on combining with equation (3.20), leads to

$$
|B|_{\mathrm{SmA}}=\frac{A \sqrt{4 \rho_{s}^{4} \xi^{2}+\rho^{2} \alpha_{4}^{2}}}{2 \rho_{s}^{2} \xi+\rho \alpha_{4}+2 \rho_{s} \sqrt{\rho \xi \alpha_{4}}},
$$

as well as the displacement of the refracted wave, taken in the form

$$
\boldsymbol{u}^{\mathrm{SmA}}=\left(C_{\mathrm{SmA}} \exp \{i[\omega t-k(y \sin \phi+q z)]\}, 0,0\right),
$$

which, by appeal to equations (3.18) and (5.1), yields

$$
|C|_{\mathrm{SmA}}=\frac{2 A \rho_{s} \sqrt{2 \xi}}{\sqrt{2 \rho_{s}^{2} \xi+\rho \alpha_{4}+2 \rho_{s} \sqrt{\rho \xi \alpha_{4}}}} .
$$

This, combined with (3.18) and (5.3), provides a full description of the refracted wave in the SmA in terms of the prescribed parameters $A, \omega, k$ and $\phi$ along with the SmA material parameters $\rho$ and $\alpha_{4}$. 


\section{Smectic C}

In a similar manner, the analogous quantities in the $\mathrm{SmC}$ are

$$
\begin{aligned}
|B|_{\mathrm{SmC}} & =\frac{A \sqrt{4 \rho_{s}^{4} \xi^{2} \lambda_{5}^{2}+\rho^{2} \Gamma_{1}^{2}}}{2 \rho_{s}^{2} \xi \lambda_{5}+\rho \Gamma_{1}+2 \rho_{s} \sqrt{\rho \xi \lambda_{5} \Gamma_{1}}}, \\
|C|_{\mathrm{SmC}}= & \frac{2 A \rho_{s} \sqrt{2 \xi \lambda_{5}}}{\sqrt{2 \rho_{s}^{2} \xi \lambda_{5}+\rho \Gamma_{1}+2 \rho_{s} \sqrt{\rho \xi \lambda_{5} \Gamma_{1}}}},
\end{aligned}
$$

recalling that, in (5.5), we are displaying the behaviour of mode 2 for the reasons outlined above in Section 4.

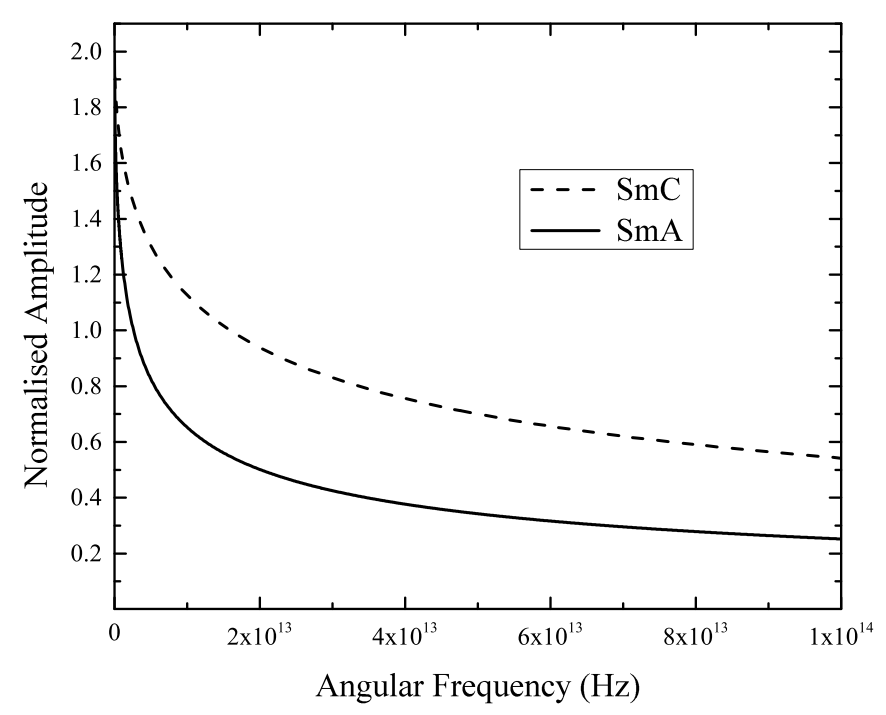

Figure 4: The amplitudes $|C|_{\mathrm{SmA}}$ and $|C|_{\mathrm{SmC}}$ of the refracted waves, normalised with respect to $A$. Note that both continuum theories predict the same qualitative behaviour.

Figure 4 displays the behaviour of the amplitudes in both the $\mathrm{SmA}$ and $\mathrm{SmC}$, i.e. $|C|_{\mathrm{SmA}}$ and $|C|_{\mathrm{SmC}}$, as functions of the incident angular frequency $\omega$. Parameter values for SmC were taken from [12, p. 301] where we have set $\tau_{5}-\tau_{1}>0, \lambda_{5}-\lambda_{2}>0$ and $\theta=\pi / 6$. The values for SmA were obtained from Table 1 in reference [15]; for the solid, we chose $\rho_{s}=2400 \mathrm{kgm}^{-3}$ and $\mu_{s}=2.62 \times 10^{10} \mathrm{~Pa}$. Clearly the behaviour of each of these expressions in (5.3) and (5.5) is qualitatively the same, and we observe that, until $\omega \sim 10^{10} \mathrm{~Hz}$,

$$
\begin{aligned}
|C|_{\mathrm{SmA}} & \sim\left(2-1.01 \times 10^{-6} \sqrt{\omega}\right) A, \\
|C|_{\mathrm{SmC}} & \sim\left(2-4.12 \times 10^{-7} \sqrt{\omega}\right) A,
\end{aligned}
$$

from which it is evident that $|C|_{\mathrm{SmA}} \doteqdot 1.9 A$ and $|C|_{\mathrm{SmC}} \doteqdot 1.96 A$ when $\omega=10^{10} \mathrm{~Hz}$, both showing comparatively little change over the range $0 \leq \omega \lesssim 10^{10} \mathrm{~Hz}$. Thereafter, the refracted wave amplitudes begin to fall off more noticeably, and by $\omega=10^{14} \mathrm{~Hz},|C|_{\mathrm{SmA}}$ is just over one tenth of its initial value, while $|C|_{\mathrm{SmC}}$ is somewhat below thirty percent of its initial value.

\section{Discussion}

Results have been presented regarding the behaviour of a shear wave at the interface between a solid and an unbounded sample of SmA liquid crystal, fully establishing the behaviour of the reflected 
and refracted waves in terms of the problem's physical parameters. In particular, the refracted wave number $q$, which characterises the attenuation of the wave in the $\mathrm{SmA}$ case, was provided in terms of the parameters characterising the solid and the smectic by equation (3.16). Further, expressions for the amplitudes of these at normal incidence were derived in terms of the incident wave amplitude and these parameters, with (5.2) and (5.3), lead to expressions for the reflected and refracted waves, respectively. For the purpose of a qualitative comparison, we derived analogous terms via the results of Gill and Leslie [2], who performed calculations for the identical experiment for a sample of SmC, utilising the LSN theory for SmC. It is readily seen that, at normal incidence, the behaviour of the two phases is qualitatively the same, with the refracted wave amplitudes showing a departure in behaviour from the approximate expressions given in equations (5.6) and (5.7) as $\omega$ increases beyond the critical value $10^{10} \mathrm{~Hz}$. Before $\omega$ attains this value, the aforementioned expressions provide a very accurate approximation to the respective exact expressions for the refracted wave amplitudes given in (5.3) and (5.5).

The assumption of spatially semi-infinite samples is valid for samples whose depth is greater than that of the penetration depth given in either (4.18) for $\mathrm{SmC}$ or (4.19) for $\mathrm{SmA}$ in the case of normal incidence. It may prove instructive to consider a smectic confined to a region whose depth is less than that of these penetration depths and investigate the effects of the boundaries in this case. Further, the linear stability of the layered structure to perturbations will presumably be valid for sufficiently small amplitudes of incident wave; just how sensitive the smectic sample is to higher magnitude disturbances is a matter for further investigation.

\section{References}

[1] Landau, L. D., Lifshitz, E. M. (1987). Fluid Mechanics, Landau and Lifshitz: Course of Theoretical Physics, Vol. 6, 2nd Ed., Butterowrth-Heinemann: Oxford, UK.

[2] Gill, S. P. A., Leslie, F. M. (1992). J. Mech. Phys. Solids, 40, 1485.

[3] Stewart, I. W. (2007). Continuum Mech. Thermodyn., 18, 343.

[4] Auernhammer, G. K., Brand, H. R., Pleiner, H. (2000). Rheol Acta, 39, 215.

[5] Auernhammer, G.K., Brand, H.R., Pleiner, H. (2002). Phys. Rev. E, 66, 061707.

[6] Ribotta, R., Durand, G. (1977). J. Physique, 38, 179.

[7] Aris, R. (1989). Vectors, Tensors, and the Basic Equations of Fluid Mechanics, Dover: New York, USA.

[8] De Vita, R., Stewart, I. W. (2013). Soft Matter, 9, 2056.

[9] Landau, L. D., Lifshitz, E. M. (1986). Theory of Elasticity, Landau and Lifshitz: Course of Theoretical Physics, Vol. 7, 3rd Ed., Butterowrth-Heinemann: Oxford, UK.

[10] Spencer, A. J. M. (2004). Continuum Mechanics, Dover: New York, USA.

[11] Leslie, F. M., Stewart, I. W., Nakagawa, M. (1991). Mol. Cryst. Liq. Cryst., 198, 443.

[12] Stewart, I.W. (2004). The Static and Dynamic Continuum Theory of Liquid Crystals, Taylor \& Francis: London, UK.

[13] Gill, S. P. A. (1992). Ph.D. Thesis, Department of Mathematics, University of Strathclyde, Glasgow.

[14] Miscandlon, J., Stewart, I. W. In preparation.

[15] Stewart, I.W., Stewart, F. (2009). J. Phys. Condens. Matter, 465, 101. 